\title{
El ejemplo y la experiencia de las comunidades de práctica como aportadoras de conocimiento especializado en la educación patrimonial
}

\author{
The example and experience of the communities of practice: \\ feeding in expertise in the field of heritage education
}

\section{Maria Cacheda*}

\footnotetext{
* Agència Catalana del Patrimoni Cultural, Generalitat de Catalunya, Departament de Cultura. Licenciada en Historia del Arte con estudios de doctorado y DEA en Arqueología, Historia de la Antigüedad y Ciencias y Técnicas Historiográficas. Coordinadora de la unidad de Acción Educativa de la Agencia Catalana del Patrimonio Cultural del Gobierno de Cataluña, e investigadora del grupo Centro de Estudios del Patrimonio Arqueológico de la Prehistoria (CEPAP) de la Universidad Autónoma de Barcelona.

$\triangle$ mcacheda@gencat.cat
}

\begin{abstract}
Resumen
La educación, como práctica de transmisión y creación de saberes, y la autoformación para la construcción de algo común son la misión principal y los valores que agrupan a personas con ganas de cambiar la irreversibilidad imperante en la práctica diaria. Estas personas, que nos dedicamos a la educación patrimonial, nos hemos juntado en comunidades de práctica para poder expresarnos y trabajar con libertad y autonomía, en horizontalidad y con el compromiso de mejora de nuestra práctica profesional.
\end{abstract}

Palabras clave: patrimonio cultural, educación, España.

\begin{abstract}
Education, understood as transmission and creation of knowledge, and self-training to build commonalities, are the main objectives and values shared by those willing to change the prevailing irreversibility in daily practice. People dedicated to heritage education have come together in communities of practice to express ourselves and work with freedom and autonomy, based on principles of horizontality and with the commitment to improve our professional practice.
\end{abstract}

Keywords: cultural heritage, education, Spain. 


\begin{abstract}
At the beginning of 2017, the closing down of FANAPEL (National Paper Factory), founded in 1898, shook the city of Juan Lacaze, calling the attention of government and media. The political strategies developed by this company's workers, alongside with other organized sectors of the city, were fundamental to place this issue on the public agenda. This essay, based on the testimony of professionals working in the area, addresses the trajectories of subjects linked to two different sectors of the city: the rural and the vulnerable, away from highly politicized scenes, from an anthropological perspective. The general objective, then, is to increase their visibility, in the context of the construction of a development agenda coordinated by the Local Development program of CLAEH University.
\end{abstract}

Keywords: participatory development, civil society, rural population, disadvantaged groups.

\title{
Introducción
}

La Agencia Catalana del Patrimonio Cultural (ACdPC) nace en 2014 con el objetivo primero de ayudar y contribuir, de una manera favorable y justa, a la gestión y coordinación de las instituciones patrimoniales y los museos que pertenecen al gobierno de Cataluña, la Generalitat de Catalunya.

La ACdPC tiene por misión gestionar, con relación al medio urbano y natural que lo acoge, el patrimonio cultural de la Generalitat con criterios de integridad, sostenibilidad y eficiencia. Además, la Agencia apoya al departamento competente en materia de cultura en las actividades programadas para ejecutar las políticas establecidas por la unidad correspondiente en materia de patrimonio cultural. La Agencia tiene adscritos treinta y ocho monumentos, cinco museos y un centro cultural de restauración de bienes muebles.

Las funciones, entre otras, que realiza la ACdPC son:

- Dinamizar el patrimonio cultural mediante la cooperación entre agentes públicos y privados para mejorar la promoción, la conservación y la gestión, especialmente estableciendo estrategias de apoyo y colaboración con los entes locales.

- Fomentar la vinculación del patrimonio cultural con el desarrollo territorial y paisajístico y con el desarrollo económico, con el fin de generar nuevos recursos destinados a la promoción y conservación de este patrimonio.

- Fomentar el uso del patrimonio cultural y de las instituciones, prestando especial atención a la educación y el turismo cultural en colaboración con el departamento 
competente en materia de turismo; mejorar la vinculación con la comunidad y la internacionalización, y fomentar las prácticas asociadas a la conservación y divulgación de este patrimonio.

- Prestar servicios de restauración, conservación, rehabilitación, excavación arqueológica, mejora de la calidad y puesta en valor de bienes culturales y de divulgación y de información en materia de patrimonio cultural.

- Promover la formación, la investigación y la transferencia de conocimiento en todos los ámbitos del patrimonio cultural, y la incorporación de las tecnologías de la información y la comunicación en la gestión y la información en materia de patrimonio cultural.

- Gestionar la red de monumentos y yacimientos arqueológicos de titularidad de la Generalitat y los equipamientos patrimoniales adscritos al departamento competente en materia de cultura.

- Facilitar la participación de la ciudadanía en la gestión y la promoción del patrimonio cultural, incluyendo en este ámbito el fomento del patrocinio, del mecenazgo y del voluntariado.

Concretamente, desde la perspectiva de la acción educativa, que nace y se impulsa a partir de los inicios de 2015, se entiende como misión seguir y acompañar la transformación educativa que se está generando en el sistema educativo formal catalán, por un lado, con la renovación de las metodologías pedagógicas y, por otro, impulsando formaciones y seminarios para profundizar en la experiencia de los y las profesionales de la educación patrimonial. Investigar, estudiar y buscar nuevas maneras de proceder en el conjunto de los programas educativos de museos y monumentos es fundamental para que el aprendizaje que se promueva hacia los visitantes sea significativo y que el alumnado se sienta protagonista de estas experiencias. Tenemos la necesidad de superar el modelo de enseñanza transmisor y posibilitar que los niños, niñas y jóvenes puedan disfrutar en el museo de experiencias de aprendizaje relevantes, que los ayuden a ser personas autónomas y críticas, y también que ello revierta en una sociedad más cohesionada, capaz de dar respuesta a los retos globales, formando ciudadanos libres y responsables.

Los centros patrimoniales, normalmente con pocos recursos humanos y económicos, se encuentran con el reto de mejorar las pedagogías de sus acciones educativas. Partimos del convencimiento de que la educación mejora a través de la colaboración, el intercambio y el aprendizaje mutuo. Un acercamiento entre museo y escuela sería conveniente para preguntarnos qué es lo que podemos ofrecer y aportar, de modo que estas acciones educativas dejen de valorarse, en muchos casos, como un producto de consumo. Los cambios que se reclaman en la escuela son también necesarios en los museos. Necesitamos pedagogías adaptadas al siglo XXI (Cacheda, 2017). 
Parafraseando a la filósofa Marina Garcés (2015), que pase algo en nuestra vida, y por lo tanto también en el trabajo que hacemos, tiene que ver con tres potencialidades creativas que todos y todas tenemos en nuestro interior: abrir, significar y decidir. Abrir posibilidades que antes no existían: por ello, entre un grupo de personas interesadas en este cambio educativo hemos creado la Comunidad de Práctica Patrimonio y Escuela (CoP Patrimonio y Escuela) y la Comunidad de Práctica virtual Guarderías y Patrimonio, con la intención de producir significados nuevos, tomando la decisión y el compromiso conjunto de transformar en clave competencial la acción educativa del patrimonio, rompiendo así la irreversibilidad imperante general en la educación patrimonial.

Nuestra microrrevolución colectiva es constituirnos en comunidades de práctica (CoPs) a partir de la voluntariedad de un conjunto de personas inquietas, profesionales activas en educación formal e informal, que creemos en el cambio mediante la implicación personal y cooperativa.

\section{Las comunidades de práctica (CoP)}

¿Pero qué es esto de una CoP? Las CoP son grupos de personas de un colectivo concreto, asociadas de manera voluntaria con el objetivo de desarrollar un conocimiento especializado mediante la colaboración entre ellas, compartiendo experiencias, información y conocimientos de manera estructurada y limitada en el tiempo (Vázquez Bronfman, 2011). Se trata de una herramienta de gestión del conocimiento que traduce estos saberes compartidos en un producto de conocimiento aplicable a la práctica diaria del colectivo implicado (Esteve y Carandell, s.f.). Se entiende como producto de conocimiento la materialización física del objetivo trabajado en el grupo. Las CoP tratan carencias comunes que se plantean en el día a día del trabajo de estas personas. Tiene unos objetivos concretos, una metodología de funcionamiento y un plazo de ejecución, todo fijado por las personas de la comunidad. El reto es compartir práctica y generar conocimiento traducido en un manual, un dosier, un protocolo, una actividad concreta, etcétera.

Están formadas por un grupo impulsor, un grupo activo y un grupo periférico. El grupo impulsor está integrado por personas que creen en la transformación y la mejora de lo que hacen. Exponen sus inquietudes sobre un tema en común y las comparten con otras personas que se quieran sumar. El grupo activo son las personas que se añaden al grupo impulsor y trabajan en el interés común, profundizando en ese conocimiento a través de una interacción continuada. Y, por último, el grupo periférico es el que se beneficiará del trabajo de la CoP en cuestión, lo que repercutirá también en su práctica profesional. Esta estructura y diferentes niveles de participación facilitan que se comparta el conocimiento y se lo haga circular («Comunitats de Pràctica», s.f.). 
Es muy importante entender que una CoP no es simplemente un grupo de trabajo que se reúne por intereses afines. En una CoP, las personas se centran en la mejora de su propia actividad, a partir de la focalización de objetivos comunes de mejora o desarrollo. Pasar de la mirada formal a la mirada científica no es algo espontáneo ni fácil; llega después de un compromiso y un esfuerzo que parten del análisis y la comprensión de las experiencias y del conocimiento más informal. Todo se da a lo largo de un camino más cognitivo, para que se convierta en especializado y científico, siempre desde la vertiente de su aplicabilidad y la mejora de la práctica propia (Sanz Martos, 2013).

La clave para que estas comunidades se den y funcionen está en las personas. El compromiso adquirido de manera voluntaria, horizontal y cooperativa hace que todas y todos los que participan ganen en relaciones interpersonales, autoformación, empoderamiento y, lo más importante, la pertenencia a un grupo que será partícipe de una solución compartida y valiosa.

\section{La CoP Patrimonio y Escuela}

En Cataluña tenemos como referentes del trabajo en CoPs al Departamento de Justicia y sobre todo a la Agencia de Salud Pública, los dos organismos pertenecientes al gobierno de Cataluña que desde hace más de diez años fomentan la práctica profesional en comunidades de práctica dentro de sus proyectos anuales («Entrevista amb...», 2017). Impulsan el uso de las herramientas que permite la sociedad de la información y el conocimiento, a fin de promover la mejora continua, la eficiencia en el uso de los recursos y el aprendizaje individual y organizacional. Valorando el conocimiento de los profesionales, publican en su web una decena de trabajos hechos, pensados y elaborados por trabajadoras y trabajadores del sector en estas comunidades, con el objetivo de mejorar su práctica profesional y enriquecerla.

En la ACdPC nos fijamos en su trayectoria, la seguimos y nos entrevistamos con personas que estaban detrás de estos proyectos. Después de estos encuentros y un periodo de investigación en este campo, quisimos iniciarnos en el mundo de las CoP. Nuestra idea era juntar gente interesada del mundo educativo formal e informal. La ACdPC podía contactar con profesionales de los museos catalanes y, gracias a la colaboración del Departamento de Educación del Gobierno de Cataluña, mediante un convenio que tenemos firmado desde finales de 2014 (acuerdo marco), llegamos a los docentes.

Para mejorar la comunicación y la colaboración entre la escuela y el patrimonio, una de las propuestas de la ACdPC, bajo el paraguas de este convenio, es la Jornada de Patrimonio y Escuela. En estas jornadas dirigidas a docentes y profesionales de la educación patrimonial, la ACdPC, como grupo impulsor de la CoP, lanzó la idea de formar una de estas comunidades, ante la preocupación generalizada del sector por encontrar 
un lugar compartido de trabajo para aquellas personas preocupadas por mejorar la práctica educativa en sus instituciones patrimoniales. A través de un formulario de solicitud, una treintena de personas se interesaron y se formó finalmente un grupo activo de veinticinco miembros que trabajamos desde enero de 2017.

La novedad de esta CoP respecto a las ya formadas en otros campos es que en ella se unen dos colectivos diferentes pero provenientes del mundo educativo: docentes y profesionales de la educación de centros patrimoniales y museos, con inquietudes educativas comunes, pero con pocos espacios de interacción y de cooperación. La pertenencia a este grupo implica voluntariedad, confianza y compromiso con todos los miembros, compartir información y experiencias que permitan nuevas formas de actuación en la praxis diaria.

El objetivo de la CoP es crear unos indicadores cualitativos y compartidos para diseñar y evaluar las actividades educativas de las instituciones patrimoniales dentro de los criterios del Modelo Orientador Competencial que promueve el Departamento de Educación del Gobierno de Cataluña, para mejorar la docencia en los centros educativos. Este modelo de orientación educativa contiene los principios pedagógicos competenciales y las dinámicas organizativas y de relación de un centro educativo, orientadas a favorecer el éxito del alumnado en cuanto a su autonomía y el desarrollo de su proyecto de vida.

La metodología de trabajo que compartimos consiste en reuniones periódicas (fijadas por el grupo) y, a la vez, otros encuentros de los cuatro subgrupos en los que nos hemos subdividido para trabajar cada uno de los criterios en que se basan los cuatro pilares de aprendizaje del alumnado: significatividad, comunicación, acción y proyección. Además, entre reuniones compartimos, reflexionamos y cuestionamos el trabajo realizado a través de herramientas digitales en línea que nos mantienen activos entre las sesiones presenciales.

Según este modelo pedagógico, la significatividad se refiere a todo aquello que hacen los jóvenes y niños en todos los momentos y espacios de aprendizaje de manera que capte su interés (me intereso, me implico y gestiono, encuentro sentido a lo que hago, me doy cuenta de cómo lo hago y cómo lo aprendo). La acción tiene que ver con el alumnado como sujeto protagonista de su aprendizaje (por ajustarme al entorno cambiante debo actuar, ser activo). La proyección significa que el aprendizaje del alumnado tiene proyección, aporta una consecuencia fuera del entorno escolar, en su conexión con lo que se hace y aprende en otros ámbitos. Y la comunicación sería la herramienta de construcción y evaluación de los aprendizajes, las diferentes formas de expresión, teniendo en cuenta la diversidad del alumnado y las inteligencias múltiples.

Este modelo competencial concreta los criterios y los indicadores de la acción docente y educativa que orienta hacia el éxito del alumno, con dos significados: el éxito entendido como conexión del aprendizaje del alumnado dentro del entorno escolar con 
el entorno no escolar, y el éxito entendido como formación para la autonomía del alumnado. Ambos significados recogen dos aspectos fundamentales: la proyección de futuro de jóvenes, niños y niñas, y su autonomía.

Este prototipo se llama competencial orientador porque aglutina un conjunto de pautas metodológicas, didácticas y pedagógicas favorecedoras del proceso de aprendizaje del alumnado. Además, estas pautas reclaman un estilo docente concreto, orientado a promover este aprendizaje.

Los referentes pedagógicos en los que se basan son las teorías del aprendizaje que tapizan la tradición pedagógica del sistema educativo catalán y se insertan en el planteamiento y el diseño competencial de los currículos educativos en Cataluña. Además, la evaluación de las acciones será el referente principal para la medida y la ponderación de la capacidad de avance y de mejora de un centro educativo y, por consiguiente, de una institución cultural como un museo.

Estos indicadores en los que trabaja la CoP los compartiremos con toda la comunidad educativa para que sean utilizados y aplicados una vez que el trabajo esté terminado. La fecha de finalización prevista es entre junio y julio de 2018.

La clave del funcionamiento de esta $\mathrm{CoP}$ radica en quienes la integramos, que nos sentimos parte de un grupo de gente muy valiosa, que tiene las mismas inquietudes y curiosidades para mejorar la educación patrimonial tradicional que se da en muchos museos y centros patrimoniales. Romper la irreversibilidad de lo que persiste es un compromiso compartido en el que depositamos nuestra energía y entusiasmo. Nuestra CoP es una unidad autónoma que funciona fuera del horario laboral y con los recursos propios de cada persona participante.

Por eso, a las personas que trabajan de esta manera innovadora nos dicen coloquialmente cacahuetes, ya que al igual que la leguminosa, que es la única que da el fruto bajo tierra, nuestro grupo trabaja de manera poco visible para mejorar aspectos concretos de nuestra tarea. Hacemos un trabajo productivo y muy aprovechable como el que hace la planta del cacahuete.

Las y los cacahuetes que trabajamos en el grupo activo y en las instituciones que representamos en la CoP Patrimonio y Escuela somos: Albert Vicedo, Departamento de Educación, Gobierno d Cataluña; Andrea Granell, experta externa y colaboradora de Acción Educativa, ACdPC; Anna Catà, Auriga Servicios Culturales; Anna Plans, Museo Palau Mercader, Ayuntamiento de Cornellà de Llobregat; Carme Gilabert, Ecomuseo - Farinera de Castelló d'Empúries; Carmina Borbonet, Museo del Diseño de Barcelona; Clara López, coordinadora de la Red de Museos de las Tierras de Lleida y Aran, educadora de museos; Cristina Puig, Museo Marítimo de Barcelona; Esther Fuertes, Museo Nacional de Arte de Cataluña; Greta Boix, Museo de Ciencias Naturales de Barcelona, Nusos actividades científicas y culturales; Griselda Aixelà, Museo de Historia de Cataluña; Guido Ramellini, Museo de Matemáticas de Cataluña, Cornellà de Llobregat; Isabel de la Fuente, IES Leonardo 
da Vinci Sant Cugat; Mar Sánchez, Espacio Faro Vilanova i la Geltrú; Maria Cacheda, Acción Educativa, ACdPC; Maria Feliu, Museo de Historia de Cataluña y Universidad de Barcelona; Mireia Bassols, profesional de la educación y arterapeuta; Pere Viladot, experto externo, asesor Acción Educativa de la ACdPC; Sebastiana Bote, Instituto Terra Roja de Santa Coloma de Gramenet; Susanna Tena, Escola Sant Just de Santa Coloma de Gramenet y Vàngelis Villar, Fundación Barcelona Olímpica.

\section{La e-CoP Guarderías y Patrimonio}

Cuando hacíamos la investigación sobre trabajo colaborativo en Cataluña, también supimos que el Servicio de Formación de la Subdirección General de Personal de Administración y Servicios (PAS) del Departamento de Educación trabajaba en CoP virtuales. El personal PAS de este departamento disfruta de formaciones internas voluntarias derivadas de las CoP tradicionales, que le ofrecen horas de formación certificada dentro del sistema de acreditación propio. La diferencia con las otras CoP, además de ser una metodología más virtual, es que el proyecto de trabajo se elige con anterioridad por el comité interno del servicio de formación, teniendo en cuenta las carencias del colectivo al que va dirigido.

En mayo del 2017, contactaron con la ACdPC para exponernos su idea de colaborar en una e-CoP virtual entre educadoras de guarderías, que en Cataluña tienen categoría de personal PAS, y profesionales del mundo de la educación de instituciones patrimoniales y museos. Las dos instituciones seríamos el grupo impulsor y debíamos buscar a las personas interesadas, el grupo activo.

La idea, tras una investigación de las necesidades de las educadoras de guarderías por el Departamento de Educación, era crear una serie de actividades educativas para llevar a cabo en los centros culturales y museos que participaran, adaptadas a las necesidades educativas y capacidades de los niños de cero a tres años. Todo el material elaborado se recogería en un documento con recomendaciones para el diseño de futuras actividades. Ese es el producto de conocimiento en el que se está trabajando.

Esta e-CoP funciona desde junio de 2016 y la fecha de finalización está prevista para marzo de 2018. Se trata de un modelo semipresencial. Se han hecho algunos encuentros presenciales del grupo activo, pero el trabajo colaborativo y participativo se hace de manera virtual a través de una plataforma tipo moodle del Departamento de Educación. Destacan dos figuras importantes en este modelo de trabajo: la moderadora (e-moderadora) y la experta (e-experta). La moderadora gestiona la información de la plataforma, hace propuestas de agenda y de entrega de material y anima al grupo cuando por algún motivo se atasca o no encuentra el camino. Es una figura muy importante, también presente en las CoP tradicionales, que debe estar siempre alerta para 
asegurar que el trabajo se haga. La otra figura destacada es la experta, que en este caso es una persona con un bagaje muy amplio y experiencia contrastada en el mundo de la educación patrimonial y la pedagogía.

La búsqueda de quienes participan se hizo a través de las redes sociales Facebook y Twitter, y se apuntaron dieciséis personas, entre educadoras de guarderías y profesionales de instituciones patrimoniales.

Este es el grupo de cacahuetes de la e-CoP Guarderías y Patrimonio: Rosa Carreras, Guardería L'Oreneta (Terrassa); Rosa Farré, Guardería El Rossinyol (Santa Coloma de Queralt); Anna Fenoy, Guardería L'Espurna (Manresa); Maria dels Àngels Haro, Guardería L'Airet (Barcelona); Maria Mercè Naves, Guardería Dumbo (El Prat de Llobregat); Eva Orriols, Guardería Drac (Vilanova i la Geltru); Marta Rodríguez, Guardería El Passeig (L’Hospitalet de Llobregat); Núria Socias, Guardería El Barrufet (Reus); Anna Maria Soler, Guardería Gavot (Vilanova i la Geltru); Montserrat Soler, Guardería El Sucre (Esplugues de Llobregat); Núria Vázquez, Guardería La Baldufa (Tarragona); Anna Torres, Conjunto Rupestre La Roca de los Moros de El Cogul (El Cogul); Joan Ibarz, Real Monasterio de Santes Creus (Aiguamúrcia); Vàngelis Villar, Fundación Barcelona Olímpica (Barcelona); Gema Vives, Departamento de Patrimonio Cultural (Ayuntamiento de Cornellà de Llobregat) y Montse Ballester, Museo de Ciencias Naturales (Barcelona).

\section{Conclusión}

Según la Wikipedia (2018), la serendipia es un descubrimiento casual o imprevisto hecho por un investigador en el transcurso de una investigación orientada a otros objetivos y con presupuestos teóricos diferentes. Las serendipias se producen sin planificación y se dan de manera inesperada. Según algunos autores, estos fenómenos no obedecen solo a la casualidad, sino también a una actitud que los propicia, la que se traduce en el hecho de ser personas curiosas, flexibles y sagaces. Se habla, pues, de casualidad buscada como parte importante en los procesos de innovación y creatividad. La serendipia en nuestro caso nace de la curiosidad, de la motivación de un determinado tipo de personas muy valiosas, con la necesidad de ampliar la mirada buscando un cambio en la manera de trabajar y de relacionarnos, que tiene que ver con la generosidad, la colaboración, la horizontalidad, la autoformación y la optimización de recursos. Abrir puertas para mejorar el trabajo que hacemos y resolver desafíos recurrentes en nuestro día a día es nuestra pasión y compromiso.

En palabras de Marcel Proust: La verdadera magia del descubrimiento no consiste en buscar nuevos paisajes, sino en cambiar la mirada. 


\section{Bibliografía}

CACHEDA, M. (2017). Idees per millorar l'educació als museus (projectes col-laboratius museu-escola). Ponencia presentada a las Jornades Apropa't organitzades per la Carca de Museus de les Terres de l'Ebre i Tarragona. Recuperado de http://www.jornadesapropat.cat/wp-content/uploads/2017/12/Maria-Cacheda_Idees-per-millorar-leducacio-als-museus.pdf.

«Comunitats de pràctica» (s.f.). En Wikispaces. Recuperado de https://teories-aprenentatge-aplicacionstic.wikispaces.com/4.3.+Comunitats+de+pr\%C3\%A0ctica.

«Entrevista amb... Pius Fransoy Molina» (2017). Compartim, 49. Recuperado de http://cejfe.gencat.cat/ca/publicacions/butlleti-compartim/compartim-49.

Esteve, 0 y CARANDell, Z. (s.f.). La Comunitat de Pràctica. Recuperado de http://ateneu.xtec.cat/wikiform/wikiexport/_media/materials/practica_reflexiva/eines/comunitat_de_practica.pdf.

GARCÉs, M. (2015). Fora de clase: Textos de filosofia de guerrilla. Barcelona: Arcàdia.

SANZ MARTOS, S. (2013). «Les comunitats de pràctica són tendencia». COMEN, Revista dels Estudis de Ciències de la Informació i de la Comunicació, 19. Recuperado de http://comein.uoc.edu/divulgacio/comein/ca/numero19/articles/Article-Sandra-Sanz-Martos.html.

«Serendipia» (2018). En Wikipedia. Recuperado de https://es.wikipedia.org/ wiki/Serendipia

VÁSQUEZ BRonfman, S. (2011). «Comunidades de práctica». Educar, 47(1), 51-68. 